\title{
A set of imprinted genes required for normal body growth also promotes growth of rhabdomyosarcoma cells
}

\author{
Geoffrey Rezvani', Julian C.K. Lui', Kevin M. Barnes' and Jeffrey Baron'
}

INTRODUCTION: In many normal tissues, proliferation rates decline postnatally, causing somatic growth to slow. Previous evidence suggests that this decline is due, in part, to decline in the expression of growth-promoting imprinted genes including Mest, Plagl1, Peg3, Dlk1, and Igf2. Embryonal cancers are composed of cells that maintain embryonic characteristics and proliferate rapidly in childhood. We hypothesized that the abnormal persistent rapid proliferation in embryonal cancers occurs in part because of abnormal persistent high expression of growth-promoting imprinted genes.

RESULTS: Analysis of microarray data showed elevated expression of MEST, PLAGL1, PEG3, DLK1, and IGF2 in various embryonal cancers, especially rhabdomyosarcoma, as compared to nonembryonal cancers and normal tissues. Similarly, mRNA expression, assessed by real-time PCR, of MEST, PEG3, and IGF2 in rhabdomyosarcoma cell lines was increased as compared to nonembryonal cancer cell lines. Furthermore, siRNA-mediated knockdown of MEST, PLAGL1, PEG3, and IGF2 expression inhibited proliferation in Rh30 rhabdomyosarcoma cells.

DISCUSSION: These findings suggest that the normal postnatal downregulation of growth-promoting imprinted genes fails to occur in some embryonal cancers, particularly rhabdomyosarcoma, and contributes to the persistent rapid proliferation of rhabdomyosarcoma cells and, more generally, that failure of the mechanisms responsible for normal somatic growth deceleration can promote tumorigenesis.

I

n mammals, somatic growth is rapid in embryonic and early postnatal life but decelerates with age. This decline in growth rate is due, in large part, to a decrease in the rate of cell proliferation(1-4). We recently showed evidence that the decline in proliferation is driven by an extensive genetic program that occurs coordinately in multiple tissues during early postnatal life in mice $(5,6)$. This complex program includes the down regulation of multiple growth-promoting imprinted genes, including Igf2, Plagl1, Mest, Peg3, Dlk1, Gtl2, and Slc38a4 (7). Targeted ablation of these imprinted genes in mice causes decreased body size at birth, indicating that they are required for rapid embryonic growth (8-11). However, their expression subsequently declines markedly, probably contributing to normal postnatal growth deceleration $(5,6)$.
Although Mest, Peg3, Plagl1, and Igf2 are all required for normal somatic growth in mice, the molecular functions of the gene products are quite disparate. Igf2 encodes insulinlike growth factor-II, a secreted protein that interacts with the type I insulin-like growth factor receptor to promote growth in a wide variety of cell types. Knockout of Igf2 causes mice to be small in length and weight (8). Plagll, pleiomorphic adenoma gene-like 1, encodes a $\mathrm{C} 2 \mathrm{H} 2$ zinc-finger transcription factor. Unlike the related genes Plag1 and Plagl2, which act as proto-oncogenes, Plagl1 is considered to be a candidate tumor suppressor because reduced expression has been observed in tumor cells and because overexpression in tumor cells can induce apoptosis and cell-cycle arrest (12). However, knockout of Plagl1 in mice causes intrauterine growth restriction as well as postnatal pulmonary dysfunction (13). Mest has sequence similarity to $\alpha / \beta$ fold hydrolases. Knockout of Mest in mice causes growth retardation of embryonic and extra-embryonic structures and abnormal maternal behavior (9). Peg3 encodes a protein with 12 Kruppel-type zinc finger domains and two proline-rich periodic repeat domains. There is evidence that Peg3 promotes apoptosis and has a variety of molecular actions, including effects on Bax/p53, TNF- $\alpha$, and Wnt-related pathways (14). Mice deficient in $P e g 3$ are viable but smaller than wild-type animals, and female mutants show impaired maternal behavior including abnormal nest-building and gathering of pups (10). MEST, PEG3, PLAGL1, and IGF2 all exhibit imprinting with silencing of the maternal allele and expression of the paternal allele. It has previously been observed that a disproportionate number of imprinted genes are involved in growth regulation and particularly that paternally expressed genes tend to promote body growth (15).

Embryonal cancers are composed of cells that maintain embryonic characteristics and show abnormally rapid proliferation and other malignant features. These tumors typically present in childhood and include rhabdomyosarcoma, Wilms' tumor, neuroblastoma, and retinoblastoma, which resemble embryonic muscle, renal, neural crest, and retinal tissue, respectively. Embryonal cancers are among the most common solid extra-cranial tumors of childhood, accounting for 8-10\% of childhood malignancies (16).

${ }^{1}$ Program on Developmental Endocrinology and Genetics, Eunice Kennedy Shriver National Institute of Child Health and Human Development, National Institutes of Health, Bethesda, Maryland. Correspondence: Geoffrey Rezvani (grezvani@drexelmed.edu) 
We hypothesized that failure of the genetic program responsible for normal growth deceleration may contribute to the rapid proliferation and immature characteristics observed in embryonal cancers. Because this program appears to suppress the rapid cell proliferation that normally occurs in embryonic tissues, we reasoned that failure of the program in a clone of cells would allow persistent rapid proliferation in postnatal life, thus contributing to tumorigenesis. As a first test of this hypothesis, we focused on the growth-promoting imprinted genes that are normally down regulated postnatally, asking whether these genes show persistently elevated expression in embryonal cancers. Then, to determine whether these genes are required for the rapid growth of rhabdomyosarcoma, we knocked down expression of these genes in cultured rhabdomyosarcoma cells and examined the effect on proliferation rate.

\section{RESULTS}

To determine whether imprinted genes that have been implicated in physiological growth deceleration (6) are overexpressed in embryonal cancers, we first evaluated microarray data available from an NCI database (http://home.ccr.cancer.gov/ oncology/oncogenomics/) (17) that includes embryonal tumor samples, xenografts, and cell lines. Rhabdomyosarcoma samples showed elevated expression of MEST, PEG3, PLAGL1, IGF2, and $D L K 1$ as compared to the reference RNA sample derived from seven nonembryonal human cancer cell lines and compared to nonembryonal cancers and normal tissues (including muscle and kidney) in this database (Figure 1). Wilms' tumor samples showed elevated expression of MEST and IGF2 (Figure 1). Neuroblastoma samples showed elevated expression of only DLK1 (Figure 1). Ependymoma samples showed elevated expression of IGF2 (Figure 1). SLC38A4 did not appear to be overexpressed consistently by any tumor type (data not shown), and GTL2 expression could not be assessed because it was not represented in the database.

Because the microarray analysis showed elevated expression of multiple growth-promoting imprinted genes in rhabdomyosarcoma tumor samples, xenografts, and cell lines, we decided to focus subsequent studies on this embryonal cancer. To confirm the microarray findings, we first analyzed mRNA expression of MEST, PEG3, PLAGL, and IGF2 in rhabdomyosarcoma cell lines $(n=5)$ using real-time PCR. MEST, PEG3, and IGF2
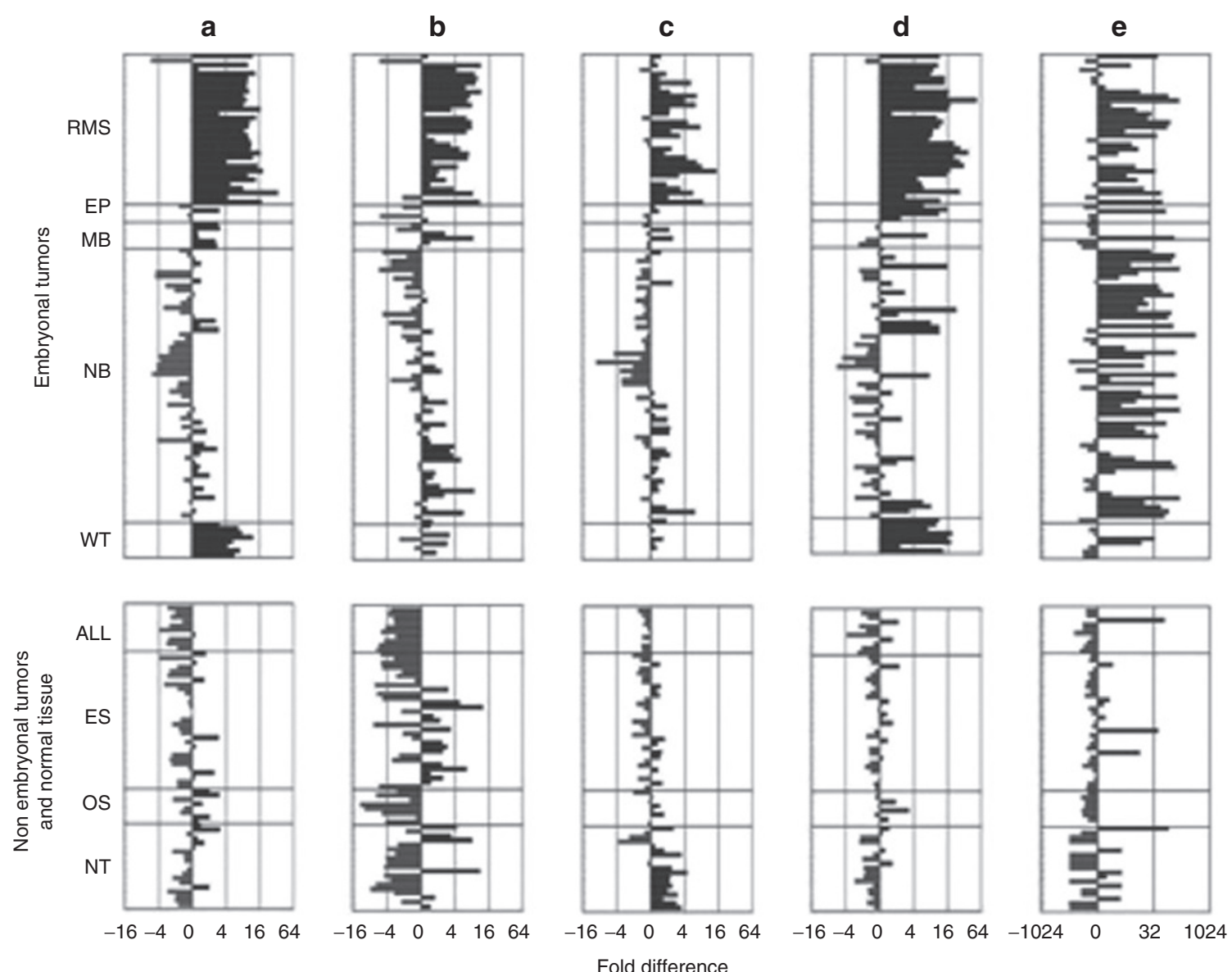

Figure 1. Expression of (a) MEST, (b) PEG3, (c) PLAGL1, (d) IGF2, and (e) DLK1 in embryonal tumors, nonembryonal tumors and normal tissues. Data are from a National Cancer Institute expression microarray database. Each bar represents a single primary tumor, xenograft, or cell line or a normal tissue sample. The plotted value is the fold difference in mRNA expression of MEST, PEG3, PLAGL1, IGF2, or DLK1 normalized to a reference RNA mixture from seven adult, nonembryonal tumor cell lines. ALL, acute lymphoblastic leukemia; EP, ependymoma; ES, Ewing's sarcoma; MB, medulloblastoma; NB, neuroblastoma; NT, normal tissue; OS, osteosarcoma; RMS, rhabdomyosarcoma; WT, Wilms' tumor. 


\section{Articles | Imprinted genes in embryonal tumors}

mRNA levels were in general higher in rhabdomyosarcoma cell lines than in nonembryonal cancers $(n=11)$ of adult and pediatric origin (Figure 2). The corresponding difference for PLAGL1 did not reach statistical significance.

To determine whether the high expression levels of these genes contribute to the rapid proliferation of rhabdomyosarcoma cells, we used siRNA interference to suppress the expression of MEST, PLAGL1, PEG3, and IGF2 in the Rh30 rhabdomyosarcoma cell line. Each gene was targeted independently with two siRNAs. Transfection of all siRNAs decreased the target mRNAs by at least $50 \%$ as compared to values from a negative control siRNA, as measured by real-time PCR (Figure 3). siRNA targeting of MEST, PEG3, PLAGL1, and IGF2 decreased proliferation as compared to values from a negative control siRNA, as assessed by ${ }^{3} \mathrm{H}$-thymidine incorporation (Figure 4 ). This effect was seen for each of the two siRNAs targeting each gene, suggesting that the effect on proliferation was not due to off-target effects of the siRNA on other genes.

Next, we analyzed the microarray data to determine whether MEST, PEG3, PLAGL1, and IGF2 are regulated coordinately in rhabdomyosarcomas. In rhabdomyosarcoma tumor samples, cell lines, and xenografts, there was a positive correlation between IGF2 expression and expression MEST, PEG3, and PLAGL1. Thus, samples that showed higher levels of IGF2 expression also tended to show higher expression levels of MEST, PEG3, and PLAGL1 (Figure 5).

Because previous evidence suggests that PLAGL1 may regulate expression of a large network of imprinted genes (13), we analyzed the effect of siRNA-mediated knockdown of PLAGL1 on the expression of MEST, PEG3, and IGF2 in Rh30 cells. PLAGL1 knockdown did not have consistent effects on mRNA levels of any of these genes (Figure 6). Similarly, knockdown of MEST, PEG3, and IGF2 had little effect on mRNA expression of any other gene studied, except for a possible reduction in PLAGL1 expression after knockdown of PEG3 (Figure 6).

\section{DISCUSSION}

We have previously shown evidence that the normal postnatal deceleration in mammalian body growth is driven by agenetic program that involves downregulation of many growth-promoting genes (5-7). Some of these downregulated growth-promoting genes are imprinted, including Igf2, Plagl1, Mest, Peg3, Dlk1,
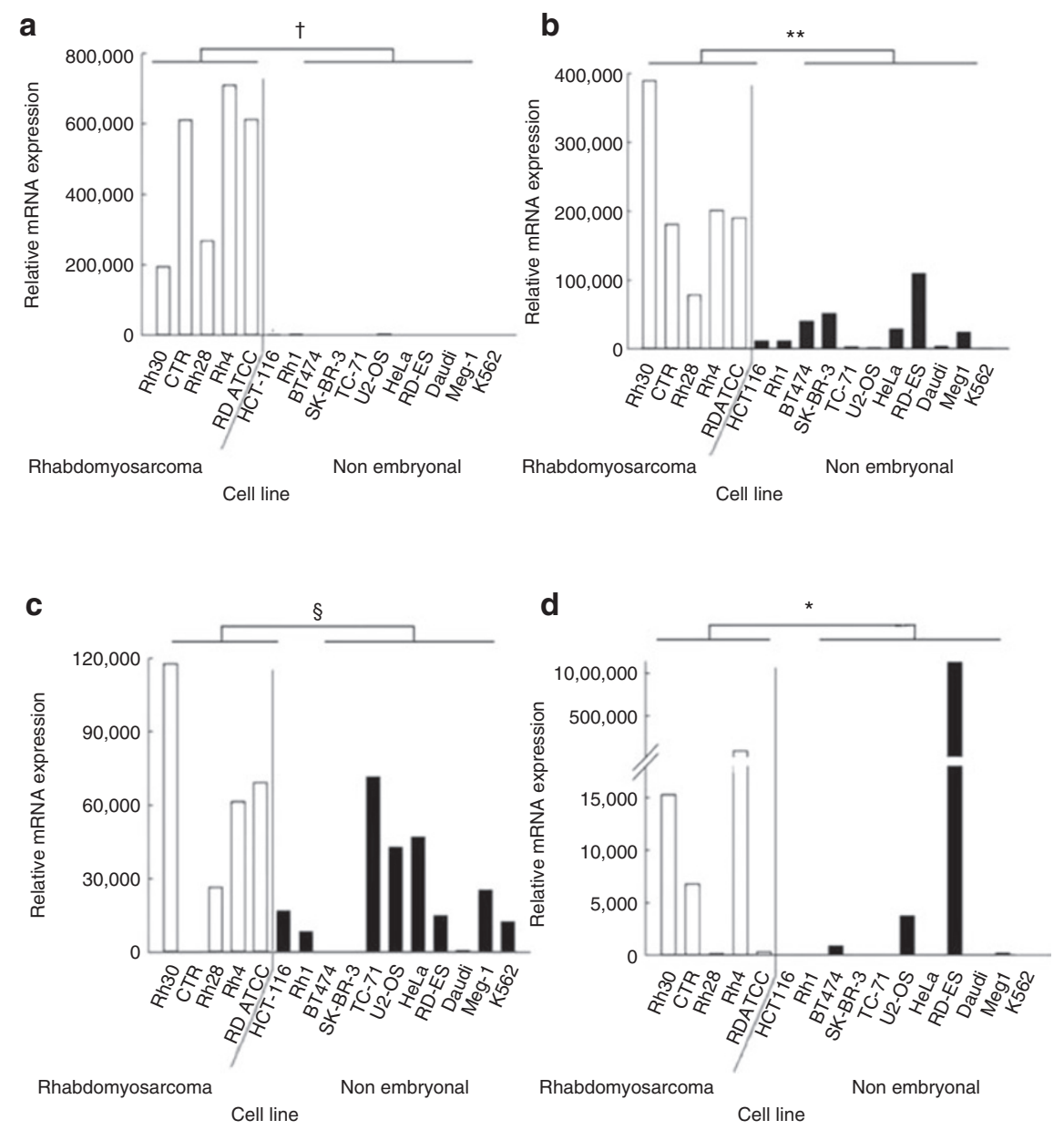

Figure 2. Relative mRNA expression of (a) IGF2, (b) MEST, (c) PLAGL1, and (d) PEG3 in rhabdomyosarcoma cell lines and nonembryonal cancer cell lines, measured by real-time PCR. Levels of IGF2, MEST, and PEG3 mRNA were significantly higher in the five rhabdomyosarcoma cell lines than in 11 nonembryonal cancer cell lines. PLAGL1 did not reach significance. White bars, relative mRNA expression in rhabdomyosarcoma cell lines; black bars, relative mRNA expression in nonembryonal cancer cell lines. ${ }^{*} P=0.05 ;{ }^{* *} P<0.005 ;{ }^{\dagger} P<0.001$; ${ }^{\S}$ nonsignificant. 


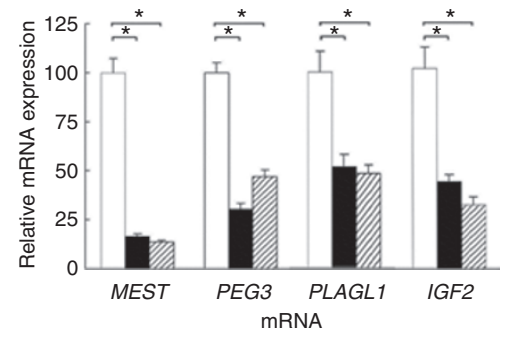

Figure 3. Effect of IGF2, MEST, PEG3, and PLAGL1 siRNA transfection on target mRNA levels in Rh30 cells. mRNA levels were measured by real-time reverse transcription PCR $48 \mathrm{~h}$ after siRNA transfection and compared to mRNA levels in cells transfected with a negative control siRNA. Two independent siRNAs were used for each gene. All siRNAs significantly reduced expression of the target gene. White bars, transfection with negative control siRNA; black bars, transfection with siRNA no. 1; hatched bars, transfection with siRNA no. $2 .{ }^{*} P<0.001$.

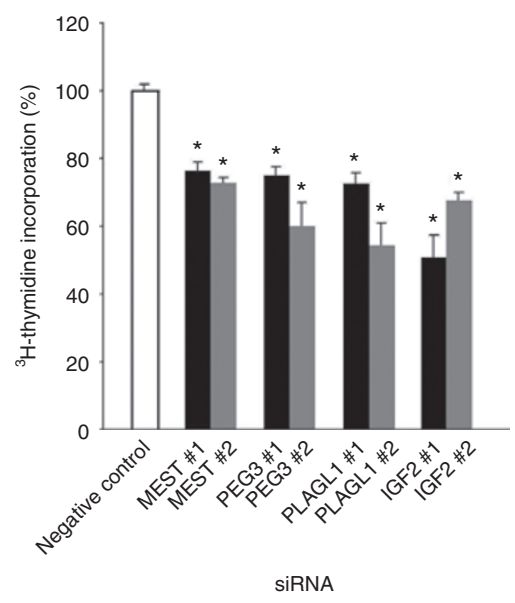

Figure 4. Effects of siRNA transfection on proliferation of Rh30 rhabdomyosarcoma cells. Cells transfected with siRNA targeting MEST, PEG3, PLAGL1, and IGF2 caused significant reduction in the rate of proliferation as compared to negative control siRNA, as measured by tritiated thymidine incorporation. This effect was confirmed with a second siRNA sequence for each gene target. White bars, transfection with negative control siRNA; black bars, transfection with siRNA no. 1; gray bars, transfection with siRNA no. $2 .{ }^{*} P<0.001$.

and Slc38a4. In this study, our analysis of available microarray data suggested that some of these imprinted genes, particularly IGF2, PLAGL1, MEST, and PEG3, are overexpressed in some embryonal cancers, especially rhabdomyosarcoma, relative to nonembryonal cancers and to normal tissues. Relative overexpression in rhabdomyosarcoma cell lines as compared to nonembryonal cancer cell lines was confirmed by real-time PCR for MEST, PEG3, and IGF2. For the analysis, tumors had to be classified as either embryonal or nonembryonal in origin. We classified Ewing's sarcoma as a nonembryonal tumor because recent molecular studies suggest that these tumors are derived from the mesenchymal stem cell (18), which is an adult stem cell present in marrow, rather than from an embryonal cell. Previous studies have focused primarily on IGF2, which has been shown to be overexpressed in some rhabdomyosarcomas (19-22), Wilms' tumors $(23,24)$, and hepatoblastomas (25). Our findings also confirm and extend a previous report that MEST, PEG3, and

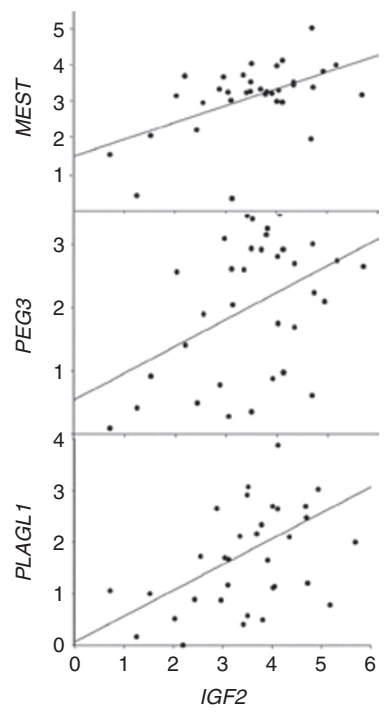

Figure 5. Correlation of expression of IGF2 with expression of MEST, PEG3, and PLAGL 1 in rhabdomyosarcoma primary tumors, cell lines, and xenografts. Plotted values are derived from a National Cancer Institute expression microarray database and represent the fold difference in expression of the mRNA as compared to a reference RNA. Samples that expressed IGF2 mRNA at high levels also expressed higher levels of MEST, PEG3, and $P L A G L 1$, suggesting that these genes are regulated coordinately in rhabdomyosarcomas. MEST: $P<0.005, r=0.497 ; P E G 3: P<0.001, r=0.617$; PLAGL1: $P<0.001, r=0.386$.
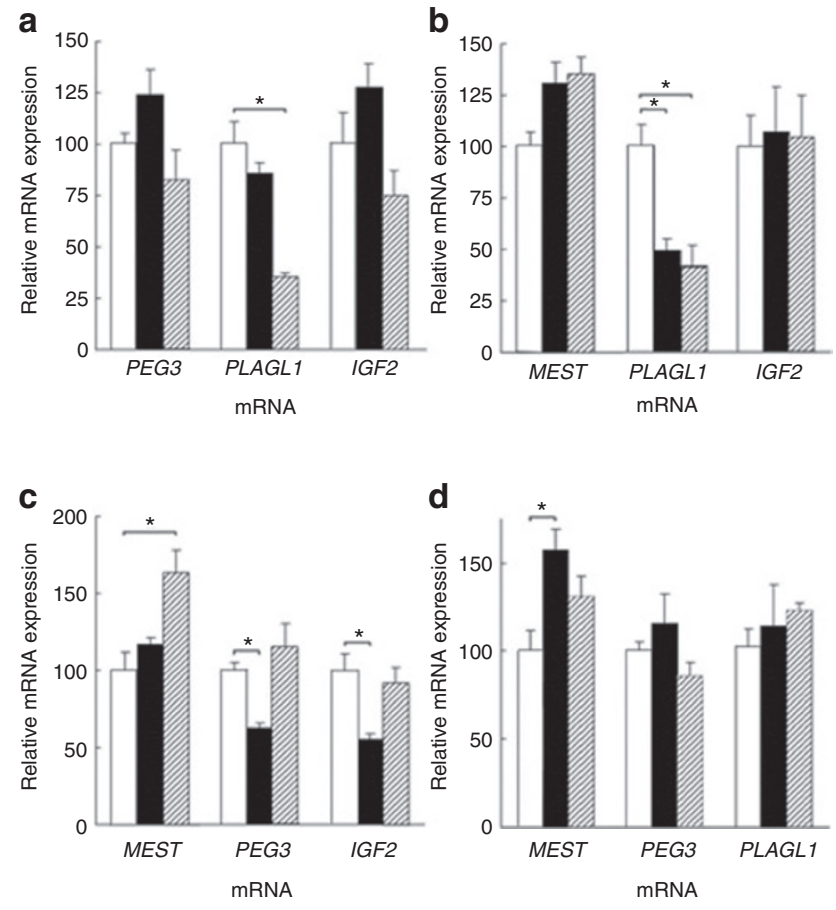

Figure 6. Effect of siRNA-mediated knockdown of (a) MEST, (b) PEG3, (c) PLAGL1, and (d) IGF2 expression on expression of other imprinted genes of interest. Rh30 rhabdomyosarcoma cells were transfected with siRNA targeting MEST, PEG3, PLAGL1, or IGF2, and the resulting effect on mRNA levels was assessed by real-time reverse transcription PCR. For each target gene, two different siRNA sequences were used independently. The only consistent effect observed was that PEG3 knockdown decreased PLAGL1 mRNA levels. White bars, transfection with negative control siRNA; black bars, transfection with siRNA no. 1; hatched bars, transfection with siRNA no. $2 .{ }^{*} P<0.05$. 
DLK1 are overexpressed in Wilms' tumors, indicating that this overexpression occurs in other embryonal cancers as well (23).

We also found that inhibiting the expression of MEST, PEG3, PLAGL1, and IGF2 slowed the proliferation of rhabdomyosarcoma cells in culture. In this experiment, we used siRNA to knock down gene expression and observed significant decreases in cell proliferation, as assessed by tritiated thymidine incorporation. Two lines of evidence suggest that the decreases in proliferation were actually mediated by decreased expression of the target gene. First, using real-time reverse transcription PCR (RT-PCR), we demonstrated that siRNA transfection did knock down the target mRNA levels by $\sim 50-80 \%$. Second, for each gene, we used two independent siRNAs and observed that both inhibited proliferation. It would be unlikely that these two siRNAs, which have different sequences, would both have off-target effects causing decreased proliferation. The results of the siRNA knockdown experiment indicate that MEST, PEG3, PLAGL1, and IGF2 are all required for the rapid proliferation observed in these cells and suggest that the observed overexpression of these genes in rhabdomyosarcomas contributes to their rapid neoplastic proliferation.

Prior studies suggest that MEST, PEG3, PLAGL1, and IGF2 are normally expressed at high levels in embryonic and early postnatal life, promoting the normal rapid body growth that occurs at this time, but are subsequently downregulated in multiple tissues simultaneously, contributing to normal somatic growth deceleration $(6,7)$. The current findings suggest that this downregulation fails to occur in some embryonal cancers and that this failure contributes to the persistent rapid proliferation of rhabdomyosarcoma cells and possibly their persistent embryonal characteristics.

Previous evidence also suggests that MEST, PEG3, PLAGL1, and IGF2 are members of an imprinted coregulated gene network (13). Consistent with this proposal, we found that MEST, PEG3, and PLAGL1 expression correlated with IGF2 expression among rhabdomyosarcoma samples. Therefore, the data suggest that these genes are regulated coordinately by a common mechanism. Varrault et al. have proposed that PLAGL1 may be a master regulator of this imprinted gene network (13). However, we found that siRNA-mediated knockdown of PLAGL1 expression in a rhabdomyosarcoma cell line did not consistently alter the expression of MEST, PEG3, or $I G F 2$. Therefore, it is unlikely that this program of declining gene expression is coordinated by PLAGL1 in rhabdomyosarcoma cells. We also tested for other possible interactions among MEST, PEG3, PLAGL1, and IGF2 expression levels and found only that knockdown of $P E G 3$ decreased expression of PLAGL1. Therefore, the findings suggest that the concordant regulation is not coordinated by any of these genes.

In conclusion, our findings provide evidence that, in addition to IGF2, other imprinted genes, including MEST, PEG3, and PLAGL1, are overexpressed in embryonal tumors including rhabdomyosarcomas and that this overexpression promotes growth of rhabdomyosarcoma cells. Our previous studies suggest that these genes are also highly expressed in embryonic cells and that the subsequent decline in expression contributes to normal growth deceleration. Therefore, taken together, the findings suggest that the postnatal program of gene expression that normally limits proliferation in juvenile tissues fails to occur in some embryonal cancers and contributes to the persistent rapid proliferation and perhaps embryonic characteristics of at least one embryonal cancer, rhabdomyosarcoma. Thus, MEST, PEG3, PLAGL1, IGF2, and DLK1 may provide a link between the genetic mechanisms responsible for normal growth deceleration and the pathologic proliferation of cells in embryonal tumors. Because the imprinted genes that we studied appear to be part of a larger genetic program responsible for growth deceleration, our findings raise the possibility that failure of other components of this program may also contribute to the growth of rhabdomyosarcoma, and possibly other embryonal cancers.

\section{METHODS}

\section{Microarray}

We analyzed an expression microarray database from the laboratory of Javed Khan (17), which is based on 163 cancer samples (75 xenograft, 70 primary tumors, and 18 cell lines), including embryonal and nonembryonal pediatric cancers. The embryonal tumor samples include rhabdomyosarcoma $(n=34)$, neuroblastoma $(n=62)$, Wilms' tumor $(n=8)$, medulloblastoma $(n=6)$, and ependymoma $(n=4)$. Cancers of nonembryonal origin include acute lymphoblastic leukemia $(n=10)$, Ewing's sarcoma $(n=31)$, and osteosarcoma $(n=8)$. Of the 163 samples analyzed in the microarray, 18 were cell lines (12 neuroblastoma, 3 rhabdomyosarcoma, and 3 Ewing's sarcoma), 70 were primary tumor samples (30 neuroblastoma, 21 rhabdomyosarcoma, and 19 Ewing's sarcoma), and the remainder were xenografts. Some of the cell lines, xenografts, and primary tumor samples might have been derived from a common origin. Normal pediatric tissue samples $(n=19)$ include gastrointestinal tissue, kidneys, ureters, muscle, uterus, testes, lung, heart, brain, and adrenal glands. Expression of mRNA was normalized to a combined sample of total RNA from seven human cancer cell lines (CHP212, RD, HeLa, A204, K562, RD-ES, and CA46). For this prior study, all samples required appropriate institutional review board and material transfer agreement approval from the donating institution (17).

\section{Cell Culture}

Rh30, Rh4, Rh28, CTR, RD-ATCC, Rh1, Daudi, K562, MEG-1, and HCT116 cell lines were cultured in RPMI 1640 (Invitrogen, Carlsbad, CA), and HeLa, BT474, SKBR3, TC-71, RD-ES, and U-2 OS cell lines were cultured in Dulbecco's modified essential medium (Invitrogen). Both culture media were supplemented with $10 \%$ fetal bovine serum (Invitrogen), $100 \mathrm{U} / \mathrm{ml}$ penicillin (Invitrogen), $100 \mathrm{U} / \mathrm{ml}$ streptomycin (Invitrogen), and $300 \mu \mathrm{g} / \mathrm{ml} \mathrm{L-glutamine} \mathrm{(Invitrogen).} \mathrm{Cells}$ were cultured at $37^{\circ} \mathrm{C}$ in $5 \% \mathrm{CO}_{2}$ and split every 3-4 days (at $\sim 75 \%$ confluence for adherent cell lines) using $0.25 \%$ trypsin with $0.038 \%$ EDTA (Invitrogen) for $1 \mathrm{~min}$. Cell lines were provided by Javed Khan, National Cancer Institute, National Institutes of Health (Duadi, K562, MEG-1, HCT116, HeLa, BT474, SKBR3, TC-71, RD-ES, U-2 OS), and Lee Helman, National Cancer Institute, National Institutes of Health (Rh30, Rh4, Rh28, CTR, RD-ATCC, Rh1). Rh30 (26-28), Rh4 (28), Rh28 (28), CTR (28), and RD-ATCC (28-30) are rhabdomyosarcoma cell lines, whereas the remaining 13 cell lines are derived from cancers of nonembryonal origin: HeLa, cervical cancer (31); HCT116, colon cancer (32); BT474 $(33,34)$ and SKBR3 (34), breast cancer; TC-71 (35), RD-ES (36), and Rh1 (26,37), Ewing's sarcoma; U-2 OS, osteosarcoma (38-40); Daudi (41), Burkitt's lymphoma; and K562 (42) and MEG-1 (43), chronic myeloid leukemia.

\section{Quantitative Real-Time RT-PCR}

Total RNA was isolated from cultured cells using the RNeasy kit (Qiagen, Valencia, CA) and then reverse transcribed using Superscript 
Table 1. siRNA sequences used for transfection experiments

\begin{tabular}{lllcc}
\hline siRNA & Sense sequence $\left(5^{\prime} \rightarrow 3^{\prime}\right)$ & Antisense sequence $\left(5^{\prime} \rightarrow 3^{\prime}\right)$ & Ambion ID no. & Concentration $(\mathrm{nmol})$ \\
\hline MEST no. 1 & GGUACAAGCAGAAUCGAUCtt & GAUCGAUUCUGCUUGUACCtg & 106797 & 15 \\
MEST no. 2 & GGGAUUGUUGUGUAGUCAtt & UUGACUACACAACAAUCCCtt & 106798 & 100 \\
PEG3 no. 1 & GCACCAGUCGAGGUCUAAAtt & UUUAGACCUCGACUGGUGCtt & 144245 & 144246 \\
PEG3 no. 2 & CGUAACUGUUCAUAAGAAUtt & AUUCUUAUGAACAGUUACGtg & 108090 \\
PLAGL1 no. 1 & GGCUUUUCCGUAAACAUAUtt & AUAUGUUUACGGAAAAGSStc & 115818 & 25 \\
PLAGL1 no. 2 & CGCGUGUUCUGUAAUCAAtt & UUUGAUUACAGAACACGCGtt & 254540 & 50 \\
IGF2 no. 1 & CAAUUGUGGAACCCACAUUtt & AAUGUGGGUUCCACAAUUGtg & 238102 \\
IGF2 no. 2 & AAUUACCUGCCCAUUCGUCtt & GACGAAUGGGCAGGUAAUUtg & 75 \\
\hline
\end{tabular}

III Reverse Transcriptase (Invitrogen), both according to the manufacturers' instructions. Quantitative real-time PCR was performed using the following assays containing primers and specific intronspanning FAM-labeled TaqMan probes (Applied Biosystems, Foster City, CA): MEST, Hs083380_91; PEG3, ZIHs00377844_m1; PLAGL1, Hs00414677; and IGF2, Hs00171254_m1. An assay for 18S rRNA using a VIC/TAMRA-labeled TaqMan probe (Applied Biosystems) was used for normalization. Reactions were performed in triplicate using cDNA, TaqMan universal PCR Master Mix (Applied Biosystems), and the ABI 7900HT Sequence Detection System (Applied Biosystems), according to the manufacturer's instructions with the following thermal cycling conditions: 1 cycle at $50^{\circ} \mathrm{C}$ for $2 \mathrm{~min}$ and $95^{\circ} \mathrm{C}$ for $10 \mathrm{~min}$, followed by $45 \mathrm{cycles}$ of $95^{\circ} \mathrm{C}$ for $15 \mathrm{~s}$ and $60^{\circ} \mathrm{C}$ for $1 \mathrm{~min}$. The quantity of each mRNA was calculated using the formula: relative expression $_{i}=(2)^{\mathrm{CT} r} /(2)^{\mathrm{CT} i}$ where $r$ represents $18 \mathrm{~S}$ rRNA (for internal normalization), $i$ represents the gene of interest, and CT represents the threshold cycle. For convenience, relative expression values were multiplied by $10^{6}$.

\section{siRNA Transfection}

One day prior to transfection, Rh30 cells were plated in 6-well plates at a density of 200,000 cells per well to reach $\sim 50 \%$ confluence at the time of transfection. Cells were transfected with siRNA (Silencer siRNA; Ambion, Austin, TX) targeting MEST, PEG3, PLAGL1, and IGF2 (Table 1), using $5 \mu$ l LipofectAMINE 2000 (Invitrogen) in $2.25 \mathrm{ml}$ total volume per well in 6-well plates. Final concentrations of siRNA required to achieve maximal knockdown were optimized. A scrambled sequence siRNA of similar length (cat. no. AM4635; Ambion) was used as a negative control at a concentration of $25 \mathrm{nmol}$. Cells were cultured at $37^{\circ} \mathrm{C}$ in $5 \% \mathrm{CO}_{2}$ for an additional $48 \mathrm{~h}$ after transfection, then used to isolate RNA for real-time RT-PCR as described previously or treated with ${ }^{3} \mathrm{H}$-thymidine as described in the following.

\section{Tritiated Thymidine Incorporation}

Cell proliferation was assessed by tritiated thymidine incorporation. Forty-eight hours after transfection with siRNA, tritiated thymidine ([Methyl- $\left.{ }^{3} \mathrm{H}\right]$ thymidine in aqueous solution, $25 \mathrm{Ci} / \mathrm{mmol}$, cat. no.TRK120; Amersham, Piscataway, NJ) was added to the culture medium at a concentration of $1 \mu \mathrm{Ci} / \mathrm{ml}$. Cells were incubated for an additional $4 \mathrm{~h}$ at $37^{\circ} \mathrm{C}$ in $5 \% \mathrm{CO}_{2}$, after which cells were detached with trypsin, and incorporation of tritiated thymidine was measured by liquid scintillation counting. For each experiment, three replicate wells were used per siRNA. The experiment was repeated, and results were combined.

\section{Statistical Analysis}

Data are presented as mean \pm SEM. Specific mRNA levels in rhabdomyosarcoma as compared to nonembryonal cancers, and effects of knockdown by siRNA on mRNA levels were evaluated by one-way ANOVA. Effects of siRNA on tritiated thymidine incorporation were normalized to results from the negative control scrambled siRNA and then evaluated by two-way ANOVA to account for effects of multiple experiments. Correlation of gene expression was evaluated by linear regression using SigmaStat (Systat Software, San Jose, CA).

\section{ACKNOWLEDGMENTS}

The authors thank Javed Khan and Lee Helman of the Pediatric Oncology Branch, National Cancer Institute, for helpful advice on the experimental approach and for providing cell lines.

\section{STATEMENT OF FINANCIAL SUPPORT}

This work was supported by the Intramural Research Program of the Eunice Kennedy Shriver National Institute of Child Health and Human Development, National Institutes of Health.

Disclosure: The authors have nothing to disclose.

\section{REFERENCES}

1 Winick M, Noble A. Quantitative changes in DNA, RNA, and protein during prenatal and postnatal growth in the rat. Dev Biol 1965;12: 451-66.

2 Chailler $\mathrm{P}$, Brière $\mathrm{N}$. Integration of proliferation and differentiation phenomena during rodent kidney ontogeny. Growth Dev Aging 1991;55:11-8

3 Post J, Hoffman J. Changes in the replication times and patterns of the liver cell during the life of the rat. Exp Cell Res 1964;36:111-23.

4 Chang M, Parker EA, Muller TJ, et al. Changes in cell-cycle kinetics responsible for limiting somatic growth in mice. Pediatr Res 2008;64:240-5.

5 Finkielstain GP, Forcinito P, Lui JC, et al. An extensive genetic program occurring during postnatal growth in multiple tissues. Endocrinology 2009; 150:1791-800.

6 Lui JC, Forcinito P, Chang M, Chen W, Barnes KM, Baron J. Coordinated postnatal down-regulation of multiple growth-promoting genes: evidence for a genetic program limiting organ growth. FASEB J 2010;24:3083-92.

7 Lui JC, Finkielstain GP, Barnes KM, Baron J. An imprinted gene network that controls mammalian somatic growth is down-regulated during postnatal growth deceleration in multiple organs. Am J Physiol Regul Integr Comp Physiol 2008;295:R189-R196.

8 DeChiara TM, Efstratiadis A, Robertson EJ. A growth-deficiency phenotype in heterozygous mice carrying an insulin-like growth factor II gene disrupted by targeting. Nature 1990;345:78-80.

9 Lefebvre L, Viville S, Barton SC, Ishino F, Keverne EB, Surani MA. Abnormal maternal behaviour and growth retardation associated with loss of the imprinted gene Mest. Nat Genet 1998;20:163-9.

10 Li L, Keverne EB, Aparicio SA, Ishino F, Barton SC, Surani MA. Regulation of maternal behavior and offspring growth by paternally expressed Peg3. Science 1999;284:330-3.

11 Gérard M, Hernandez L, Wevrick R, Stewart CL. Disruption of the mouse necdin gene results in early post-natal lethality. Nat Genet 1999;23: 199-202.

12 Van Dyck F, Declercq J, Braem CV, Van de Ven WJ. PLAG1, the prototype of the PLAG gene family: versatility in tumour development (review). Int J Oncol 2007;30:765-74. 
13 Varrault A, Gueydan C, Delalbre A, et al. Zac1 regulates an imprinted gene network critically involved in the control of embryonic growth. Dev Cell 2006;11:711-22.

14 Deng $\mathrm{Y}, \mathrm{Wu} \mathrm{X}$. Peg3/Pw1 promotes p53-mediated apoptosis by inducing Bax translocation from cytosol to mitochondria. Proc Natl Acad Sci USA 2000;97:12050-5.

15 Wood AJ, Oakey RJ. Genomic imprinting in mammals: emerging themes and established theories. PLoS Genet 2006;2:e147.

16 Paulino AC, Okcu MF. Rhabdomyosarcoma. Curr Probl Cancer 2008;32:7-34.

17 Whiteford CC, Bilke S, Greer BT, et al. Credentialing preclinical pediatric xenograft models using gene expression and tissue microarray analysis. Cancer Res 2007;67:32-40.

18 Jedlicka P. Ewing sarcoma, an enigmatic malignancy of likely progenitor cell origin, driven by transcription factor oncogenic fusions. Int J Clin Exp Pathol 2010;3:338-47.

19 Anderson J, Gordon A, McManus A, Shipley J, Pritchard-Jones K. Disruption of imprinted genes at chromosome region $11 \mathrm{p} 15.5$ in paediatric rhabdomyosarcoma. Neoplasia 1999;1:340-8.

20 Anderson J, Gordon A, Pritchard-Jones K, Shipley J. Genes, chromosomes, and rhabdomyosarcoma. Genes Chromosomes Cancer 1999;26:275-85.

21 Rikhof B, de Jong S, Suurmeijer AJ, Meijer C, van der Graaf WT. The insulin-like growth factor system and sarcomas. J Pathol 2009;217 :469-82.

22 Wada M, Seeger RC, Mizoguchi H, Koeffler HP. Maintenance of normal imprinting of H19 and IGF2 genes in neuroblastoma. Cancer Res 1995;55:3386-8.

23 Dekel B, Metsuyanim S, Schmidt-Ott KM, et al. Multiple imprinted and stemness genes provide a link between normal and tumor progenitor cells of the developing human kidney. Cancer Res 2006;66:6040-9.

24 Algar EM, St Heaps L, Darmanian A, et al. Paternally inherited submicroscopic duplication at 11p15.5 implicates insulin-like growth factor II in overgrowth and Wilms' tumorigenesis. Cancer Res 2007;67:2360-5.

25 Kim KS, Lee YI. Biallelic expression of the H19 and IGF2 genes in hepatocellular carcinoma. Cancer Lett 1997;119:143-8.

26 Hosoi H, Dilling MB, Liu LN, et al. Studies on the mechanism of resistance to rapamycin in human cancer cells. Mol Pharmacol 1998;54:815-24.

27 Cocker HA, Tiffin N, Pritchard-Jones K, Pinkerton CR, Kelland LR. In vitro prevention of the emergence of multidrug resistance in a pediatric rhabdomyosarcoma cell line. Clin Cancer Res 2001;7:3193-8.

28 Onisto M, Slongo ML, Gregnanin L, Gastaldi T, Carli M, Rosolen A. Expression and activity of vascular endothelial growth factor and metalloproteinases in alveolar and embryonal rhabdomyosarcoma cell lines. Int J Oncol 2005;27:791-8.
29 Stratton MR, Reeves BR, Cooper CS. Misidentified cell. Nature 1989;337:311-2.

30 McAllister RM, Melnyk J, Finkelstein JZ, Adams EC Jr, Gardner MB. Cultivation in vitro of cells derived from a human rhabdomyosarcoma. Cancer 1969;24:520-6.

31 Chen TR. Re-evaluation of HeLa, HeLa S3, and HEp-2 karyotypes. Cytogenet Cell Genet 1988;48:19-24.

32 Chakrabarty S, Bratain MG. The use of 125I-lectin probes in defining plasma membrane carbohydrate moieties in 3 subpopulations of human colonic carcinoma cells. Cancer Lett 1987;37:99-108.

33 Lasfargues EY, Coutinho WG, Redfield ES. Isolation of two human tumor epithelial cell lines from solid breast carcinomas. J Natl Cancer Inst 1978;61:967-78.

34 Littlewood-Evans AJ, Bilbe G, Bowler WB, et al. The osteoclast-associated protease cathepsin $\mathrm{K}$ is expressed in human breast carcinoma. Cancer Res 1997; 57:5386-90.

35 Scotlandi K, Avnet S, Benini S, et al. Expression of an IGF-I receptor dominant negative mutant induces apoptosis, inhibits tumorigenesis and enhances chemosensitivity in Ewing's sarcoma cells. Int J Cancer 2002;101:11-6.

36 Hofbauer S, Hamilton G, Theyer G, Wollmann K, Gabor F. Insulinlike growth factor-I-dependent growth and in vitro chemosensitivity of Ewing's sarcoma and peripheral primitive neuroectodermal tumour cell lines. Eur J Cancer 1993;29A:241-5.

37 Smith MA, Morton CL, Phelps D, Girtman K, Neale G, Houghton PJ. SK-NEP-1 and Rh1 are Ewing family tumor lines. Pediatr Blood Cancer 2008;50:703-6.

38 Heldin CH, Johnsson A, Wennergren S, Wernstedt C, Betsholtz C, Westermark B. A human osteosarcoma cell line secretes a growth factor structurally related to a homodimer of PDGF A-chains. Nature 1986;319:511-4.

39 Raile K, Höflich A, Kessler U, et al. Human osteosarcoma (U-2 OS) cells express both insulin-like growth factor-I (IGF-I) receptors and insulinlike growth factor-II/mannose-6-phosphate (IGF-II/M6P) receptors and synthesize IGF-II: autocrine growth stimulation by IGF-II via the IGF-I receptor. J Cell Physiol 1994;159:531-41.

40 Fogh J, Wright WC, Loveless JD. Absence of HeLa cell contamination in 169 cell lines derived from human tumors. J Natl Cancer Inst 1977;58:209-14.

41 Huber C, Sundström C, Nilsson K, Wigzell H. Surface receptors on human haematopoietic cell lines. Clin Exp Immunol 1976;25:367-76.

42 Lozzio BB, Lozzio CB. Properties and usefulness of the original K-562 human myelogenous leukemia cell line. Leuk Res 1979;3:363-70.

43 Ogura M, Morishima Y, Ohno R, et al. Establishment of a novel human megakaryoblastic leukemia cell line, MEG-01, with positive Philadelphia chromosome. Blood 1985;66:1384-92. 